\title{
Association of a missense mutation in the positional candidate gene glutamate receptor-interacting protein 1 with backfat thickness traits in pigs
}

\author{
Jae-Bong Lee, ${ }^{1, a}$, Hee-Bok Park ${ }^{2, a}$, Chae-Kyoung Yoo ${ }^{3}$, Hee-Sung Kim ${ }^{4}$, In-Cheol Cho ${ }^{2}$, and Hyun-Tae Lim,4,*
}

\footnotetext{
* Corresponding Author: Hyun-Tae Lim Tel: +82-55-772-1945, Fax: +82-55-772-1949, E-mail: s_htim@gnu.ac.kr

'Korea Zoonosis Research Institute (KoZRI), Chonbuk National University, Iksan 54531, Korea

2 Subtropical Livestock Research Institute, National Institute of Animal Science, Jeju 63242, Korea ${ }^{3}$ Institute of Agriculture and Life Science, Gyeongsang National University, Jinju 52828, Korea

${ }^{4}$ Division of Applied Life Science (BK21 plus), Gyeongsang National University, Jinju 52828, Korea

a These authors equally contributed and regarded as co-first authors.

Submitted May 30, 2016; Revised Oct 10, 2016; Accepted Dec 16, 2016
}

Objective: Previously, we reported quantitative trait loci (QTLs) affecting backfat thickness (BFT) traits on pig chromosome 5 (SW1482-SW963) in an F2 intercross population between Landrace and Korean native pigs. The aim of this study was to evaluate glutamate receptorinteracting protein 1 (GRIP1) as a positional candidate gene underlying the QTL affecting BFT traits.

Methods: Genotype and phenotype analyses were performed using the 1,105 $\mathrm{F}_{2}$ progeny. A mixed-effect linear model was used to access association between these single nucleotide polymorphism (SNP) markers and the BFT traits in the $\mathrm{F}_{2}$ intercross population.

Results: Highly significant associations of two informative SNPs (c.2442 T>C, c.3316 C>G [R1106G]) in GRIP1 with BFT traits were detected. In addition, the two SNPs were used to construct haplotypes that were also highly associated with the BFT traits.

Conclusion: The SNPs and haplotypes of the GRIP1 gene determined in this study can contribute to understand the genetic structure of BFT traits in pigs.

Keywords: Glutamate Receptor-interacting Protein 1 (GRIP1); Backfat; Quantitative Trait Loci (QTL); Landrace; Korean Native Pigs

\section{INTRODUCTION}

The pig (Sus scrofa domesticus) has been a major domestic animal species as an important animal protein source for humans and is also an excellent biomedical animal model related to obesity and energy metabolism in humans [1]. Korean native pigs (KNP) include those raised on the Korean peninsula as well as the Jeju native pigs raised on Jeju Island. Although Jeju native pigs, which are black in coat color, possess the disadvantages such as low feed efficiency, slow growth rate, and high backfat thickness (BFT), their meat is characterized by its solid fat tissue, white fat color, juiciness red meat color, and excellent marbling (Hereafter, the Jeju native pig is referred to as KNP). However, studies of the genetic factors that affect these meat quality characteristics have been limited in KNP.

Several genes are known to affect meat quality through different roles in meat quality characteristics: the ryanodine receptor 1 (RYR1) [2], liver fatty acid-binding protein ( $L-F A B P)$ [3], obesityassociated (FTO) gene [4], melanocortin receptor type 4 (MC4R) gene [5,6], POU1F1 [7-9], and protein kinase, AMP-activated and gamma 3 non-catalytic subunit (PRKAG3) [10-12].

Previously, we identified a highly significant quantitative trait loci (QTL) affecting BFT traits in pig chromosome (SSC5) [13]. This QTL region (SW1482 - SW963) includes the glutamate receptor-interacting protein 1 (GRIP1) gene. Glutamate receptor interacting protein 1 is encoded by the GRIP1 gene in human [14]. GRIP1 can directly interact with and control kinesin heavy 
chains in dendrites as a motor for the a-amino-3-hydroxy-5methylisoxazole-4-propionate (AMPA) receptor [15]. Glutamate receptor interacting protein 1 is encoded by the GRIP1 gene in human [14]. GRIP1 can directly interact with and control kinesin heavy chains in dendrites as a motor for the AMPA receptor [15]. GRIP1 plays a critical role in transmission in GABAergic and glutamatergic synapses $[16,17]$. Wu et al [18] reported that glutamatergic signaling is involved in a neuronal circuit mediating control of feeding behavior and body weight in mice [18]. Therefore, the association between single nucleotide polymorphism (SNP) markers within the exons of GRIP1 and BFT-related traits in pigs is worthy of investigation.

In this study, we presents the association of BFT traits with the GRIP1 gene using an $\mathrm{F}_{2}$ intercross population between $\mathrm{KNP}$ and Landrace pigs.

\section{MATERIALS AND METHODS}

\section{Animals and measurement of BFT traits}

All experimental processes, including breeding, feeding, and phenotype measurements, were conducted under identical conditions in the windowless house at the National Institute of Animal Science (NIAS, RDA) in Jeju Island. A three-generation resource population was produced and maintained as described by Cho et al [19]. Briefly, 19 purebred KNPs were crossed with 17 purebred Landrace. From these parental crosses, $91 \mathrm{~F}_{1}$ progeny and $1,105 \mathrm{~F}_{2}$ progeny (568 males and 537 females) were generated. None of the $\mathrm{F}_{2}$ males were castrated. The Animal Care Committee at the Jeju National University approved all procedures of experiments. From the $\mathrm{F}_{2}$ progeny, we measured BFT between the 4th and 5th ribs (BF_tho4_5), the 11th and 12th ribs (BF_tho11_12), and the last rib and first lumbar vertebrae (BF_tho_lum). The fat thickness was measured in millimeters.

\section{Polymorphism detection and genotyping}

Pools of genomic DNA samples from the parental lines (KNP $[n=5]$ and Landrace $[n=5])$ of the $F_{2}$ intercross were re-sequenced to detect SNP markers across the whole genome, including the GRIP1 gene (ENSSSCG00000000478), using the HiSeq2000 massively parallel sequencing technology (Illumina Inc., San Diego, CA, USA). We identified a total of 8 exonic SNP markers in the porcine GRIP1 gene. The 8 SNP markers were confirmed through pyrosequencing, using the primer sequences presented in Table 1. Among these, a non-synonymous SNP marker (c.3316 $\mathrm{C}>\mathrm{G}[\mathrm{R} 1106 \mathrm{G}]$ ) and synonymous SNP marker (c.2442 T>C) were found to be polymorphic in the study population. Thus, these SNP markers were genotyped in all of the $\mathrm{F}_{2}$ offspring $(n=1,105)$, using pyrosequencing (Biotage AB, Uppsala, Uppland, Sweden).

For the pyrosequencing analysis of missense and silent mutations in the GRIP1 gene, we made a primer set and minisequencing primer to be used in polymerase chain reaction (PCR) (Supplementary Table S1). Reactions were performed using $50 \mathrm{ng}$ of
Table 1. The genotype frequency and minor allele frequency (MAF) of the SNPs in GRIP1 (c.3316 C>G [R1106G], c.2442 T>C) in the an $\mathrm{F}_{2}$ intercross between Landrace and Korean native pigs

\begin{tabular}{lccccc}
\hline SNP & Region & \multicolumn{3}{c}{$\begin{array}{c}\text { Genotype frequency } \\
\text { (No. of animals) }\end{array}$} & MAF \\
\hline c.3316 C> G (R1106G) & Exon25 & CC (207) & CG (571) & GG (320) & 0.3966 \\
& & 0.1885 & 0.52 & 0.2915 & \\
c.2442 T > C & Exon20 & TT (190) & TC (544) & CC (370) & 0.3793 \\
& & 0.1721 & 0.4928 & 0.3351 & \\
\hline
\end{tabular}

SNPs, single nucleotide polymorphisms; GRIP1, glutamate receptor-interacting protein 1. ${ }^{1)}$ No. of $F_{2}$ animals genotyped.

genomic DNA, $0.4 \mu \mathrm{M}$ of each primer, $1.5 \mathrm{mM}$ of $\mathrm{MgCl}_{2}$, reaction buffer (Genetbio, Daejeon, Korea), $0.2 \mathrm{mM}$ of dNTPs, and Taq DNA polymerase (Genetbio, Daejeon, Korea) in a $25 \mu \mathrm{L}$ reaction volume. The PCR conditions were as follows: 35 cycles each of $30 \mathrm{~s}$ at $94^{\circ} \mathrm{C}$ (denaturation), $45 \mathrm{~s}$ at $63^{\circ} \mathrm{C}$ or $66^{\circ} \mathrm{C}$ (annealing), and $45 \mathrm{~s}$ at $72^{\circ} \mathrm{C}$ (elongation), 2 minutes at $94^{\circ} \mathrm{C}$ (denaturation). The genotype of the PCR products was analyzed using a pyrosequencer.

Statistical analysis of phenotype data and association test The descriptive statistical analysis and ascertainment of normality of BFT traits were conducted using MINITAB Release 14 (MINITAB Inc., State College, PA, USA). We applied a mixedeffects linear model to perform SNP association analyses between the genetic markers and traits with simultaneous consideration of familial structure in an F2 intercross between Landrace and Korean native pigs:

$$
\mathrm{Y}_{\mathrm{ijklm}}=\mu+\mathrm{S}_{\mathrm{i}}+\mathrm{B}_{\mathrm{j}}+\mathrm{P}_{\mathrm{k}}+\mathrm{G}_{1}+\mathrm{b}_{1} \mathrm{CW}_{\mathrm{ijklm}}+\mathrm{A}_{\mathrm{ijk} k \mathrm{~m}}+\varepsilon_{\mathrm{ijk} k \mathrm{~m}}
$$

Where, $\mathrm{Y}_{\mathrm{ijk} k m n}$ is the measured phenotype; $\mu$ is the population mean, $S_{i}$ is the fixed effect of ith sex, $B_{j}$ is the fixed effect of $j$ th batch, $P_{k}$ is the fixed effect of kth parity, $G_{1}$ is the fixed effect of lth genotype of GRIP1, $\mathrm{b}_{1}$ is a regression coefficient, $\mathrm{CW}_{\mathrm{ijk} / \mathrm{m}}$ is the covariate for the carcass weight, $\mathrm{A}_{\mathrm{ijk} \mathrm{km}}$ is the random additive polygenic animal effect and $\varepsilon_{\mathrm{ijk} m \mathrm{~m}}$ is the random residual effect. The mean and variance for random additive polygenic animal effects can be defined as: $\mathrm{A} \sim \mathrm{N}\left(0, A \sigma_{\mathrm{a}}^{2}\right)$, where $\mathrm{A}$ is based on the relationship matrix computed from the $\mathrm{F}_{2}$ intercross in this study and $\sigma_{\mathrm{a}}^{2}$ is the additive polygenic variance. The mean and variance for the residual random effect of individuals can be defined as: $\varepsilon \sim \mathrm{N}\left(0, I \sigma_{\mathrm{e}}^{2}\right)$, where I is the identity matrix and $\sigma_{\mathrm{e}}^{2}$ is the residual variance [20].

The significance level of the fixed effects in MODEL 1 was computed by the Wald procedure implemented in ASReml-R. The p-values of less than 0.05 were considered as statistically significant and p-values of less than 0.001 were considered as statistically highly significant. To estimate genotypic values and their standard error of each SNP genotype, the predict command in ASReml-R was used [21]. In order to estimate the additive and dominance coefficients of each SNP found in GRIP1, $\mathrm{G}_{1}$ in the 
Model 1 was replaced by additive variable $\mathrm{X}_{\text {additive }}$ (coded as 1 for rare allele homozygote, -1 for common allele homozygote, and 0 for heterozygote), and dominance variable $\mathrm{X}_{\text {dominance }}$ (coded as 0 for two homozygotes, 1 for heterozygote). Then, trait values were regressed onto the additive and dominance coefficients at each SNP marker. The PedPhased program based on the pedigree structure was used to infer the haplotypes of GRIP1 in the $\mathrm{F}_{2}$ population [22].

\section{RESULTS AND DISCUSSION}

We previously reported QTLs affecting BFT traits on SSC5 [13]. The support interval region of the QTLs estimated by the 1.5-LOD drop method was SW1482 - SW963. In this study, a positional candidate gene analysis was performed to test whether GRIP1 in the support interval region can affect BFT traits. For the descriptive statistics, the mean \pm standard error of the BFT traits were 34.02 \pm 0.24 (BF_tho4_5), 27.99 \pm 0.23 (BF_tho11_12), and 26.15 \pm 0.22 (BF_tho_lum).

\section{Search for mutation at the GRIP1 gene}

We identified 8 putative nucleotide substitutions in GRIP1 in the exon regions using HiSeq2000 massively parallel sequencing technology (Supplementary Table S1). Among these eight putative substitutions, we detected one missense substitution [c.3316 C > G (R1106G)] and seven synonymous exonic nucleotide substitutions. To conduct the association analysis, we selected c.3316 C > G (R1106G) and c.2442 $\mathrm{T}>\mathrm{C}$ because the other six substitutions were invariant, as shown in (Supplementary Table S2).

The genotyping results of c.3316 C>G (R1106G) and c.2442 $\mathrm{T}>\mathrm{C}$ obtained using pyrosequencing are shown in (Supplementary Figure S1). Data on the identified genotypes and allele frequencies of c.3316 C > G (R1106G) and c.2442 T>C in GRIP1 are presented in (Table 1). The Haploview results indicated that the alleles of the two SNPs were highly associated, so it was expected that the significant results would be very similar each other (Figure 1).

Liu and Cull-Candy [23] reported that the dynamic regulation of AMPA receptors by GRIP and protein interacting with C-kinase-1 (PICK) provide a mechanism for controlling the $\mathrm{Ca}^{2+}$ permeability of the synaptic receptors. Wu et al [18] reported that glutamatergic signaling is implicated in a neuronal circuit mediating regulation of feeding behavior and body weight in mice [18]. Hence, we investigated the association between SNP markers within the exons of GRIP1 and BFT-related traits in pigs.

\section{Association of the GRIP1 gene with BFT traits}

The BFT traits were measured in more than $1,010 \mathrm{~F}_{2}$ animals. A linear mixed-effects model accounting for familial relatedness was used to evaluate the associations between each SNP marker and the BFT traits in the $\mathrm{F}_{2}$ intercross population. These two SNP markers were significantly associated with BFT between the 4th and 5th thoracic ( $p$ value $=0.00096$ for $c .3316 \mathrm{C}>\mathrm{G}$ [R1106G]; $\mathrm{p}$ value $=0.00022$ for $\mathrm{c} .2442 \mathrm{~T}>\mathrm{C}$ ), BFT between the 11th and 12th thoracic ( $p$ value $=6.84 \times 10^{-5}$ for c.3316 C $>\mathrm{G}$ [R1106G]; $p$ value $=9.62 \times 10^{-6}$ for $\mathrm{c} .2442 \mathrm{~T}>\mathrm{C}$ ), and BFT between the last thoracic and first lumbar vertebrae ( $\mathrm{p}$ value $=7.92 \times 10^{-12}$ for $\mathrm{c} .3316$ C>G [R1106G]; $p$ value $=1.99 \times 10^{-14}$ for $\left.\mathrm{c} .2442 \mathrm{~T}>\mathrm{C}\right)($ Table 2$)$.

The highly significant two SNPs showed additive effects on BF_tho4_5 and BF_tho11_12, as no significant dominant effect was observed. In case of BF_tho_lum, however, the c.3316 C > G and c.2442 $\mathrm{T}>\mathrm{C}$ showed both additive and dominance effects. Regarding the SNP c.3316 C>G, C3316 allele was associated with higher values of BFT values. For the SNP c.2442 T>C, T2442 allele showed higher value than C2442 allele.

Following the genome-wide linkage analysis to identify QTLs for BFT traits, a positional candidate gene analysis was conducted using an intercross between KNP and Landrace pigs. We screened the GRIP1 full-length exons for polymorphisms and detected a

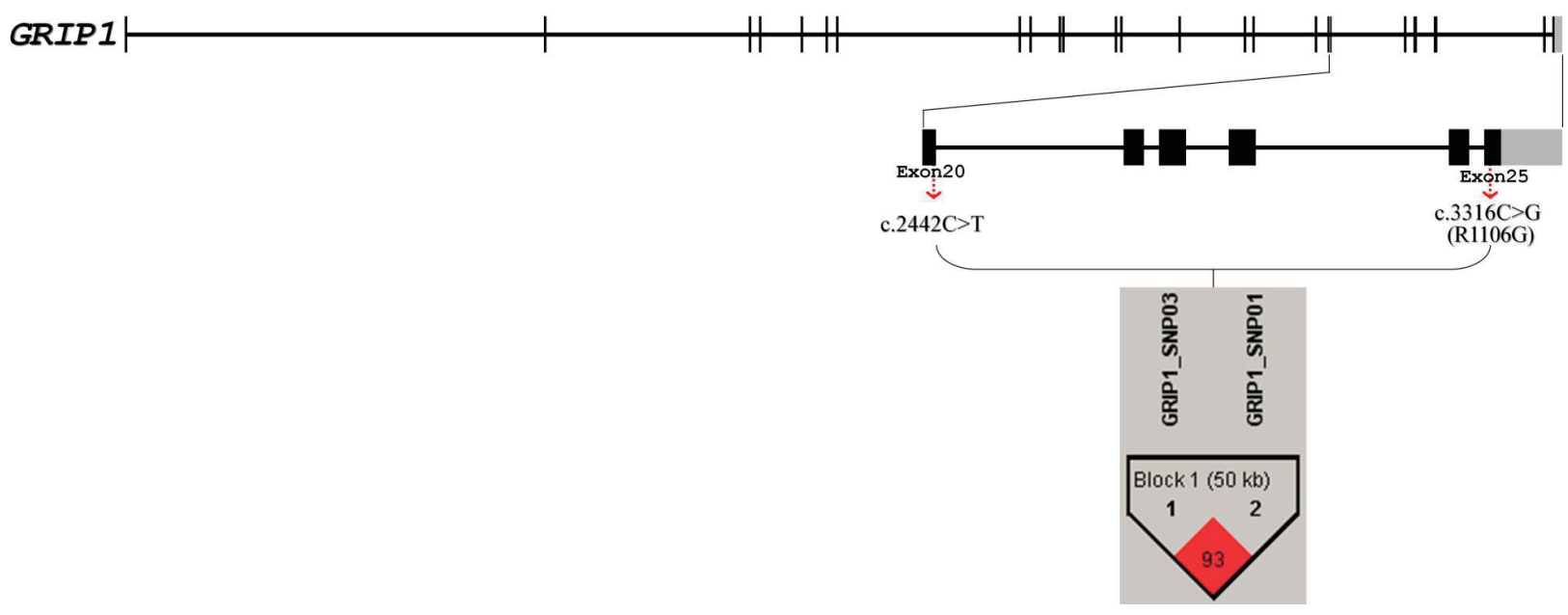

Figure 1. Exonic nucleotide variants and linkage disequilibrium (LD) block of the glutamate receptor-interacting protein 1 (GRIP1) gene (Exon 20 and 25). For haplotype analysis, LD block between single nucleotide polymorphism markers was estimated using Haploview v 4.2 software. 
Table 2. The SNP effect in GRIP1 on BFT traits in an F2 resource population between Landrace and Korean native pigs

\begin{tabular}{|c|c|c|c|c|c|c|}
\hline \multirow{2}{*}{ Traits } & \multicolumn{3}{|c|}{ c.3316 C>G (R1106G) } & \multirow{2}{*}{$p$-value } & \multirow{2}{*}{ Additive \pm SE } & \multirow{2}{*}{ Dominance $\pm S$} \\
\hline & $\mathrm{CC}(207)$ & CG(571) & GG(320) & & & \\
\hline BF_tho4_5 & $34.70 \pm 0.95$ & $33.99 \pm 0.89$ & $32.83 \pm 0.92$ & 0.00096 & $0.96 \pm 0.26$ & - \\
\hline BF_tho11_12 & $28.38 \pm 0.95$ & $27.52 \pm 0.89$ & $26.30 \pm 0.92$ & $6.84 \times 10^{-5}$ & $1.06 \pm 0.24$ & - \\
\hline \multirow[t]{3}{*}{ BF_tho_lum } & $27.81 \pm 0.92$ & $26.97 \pm 0.86$ & $24.77 \pm 0.89$ & $7.92 \times 10^{-12}$ & $1.52 \pm 0.24$ & $0.68 \pm 0.29$ \\
\hline & \multicolumn{3}{|c|}{ c. $2442 \mathrm{~T}>\mathrm{C}$} & & & \\
\hline & $\mathrm{TT}(190)$ & $\mathrm{TC}(544)$ & $\mathrm{CC}(370)$ & & & \\
\hline BF_th04_5 & $34.92 \pm 0.96$ & $34.01 \pm 0.89$ & $32.93 \pm 0.90$ & 0.00022 & $1.01 \pm 0.25$ & - \\
\hline BF_tho11_12 & $28.48 \pm 0.95$ & $27.60 \pm 0.90$ & $26.35 \pm 0.91$ & $9.62 \times 10^{-6}$ & $1.07 \pm 0.24$ & - \\
\hline BF_tho_lum & $28.14 \pm 0.91$ & $27.06 \pm 0.85$ & $24.93 \pm 0.87$ & $1.99 \times 10^{-14}$ & $1.61 \pm 0.23$ & $0.53 \pm 0.29$ \\
\hline
\end{tabular}

SNP, single nucleotide polymorphism; GRIP1, glutamate receptor-interacting protein 1; BFT, backfat thickness; SE, standard error.

novel association between the missense mutation in the GRIP1 gene (c.3316 C>G [R1106G]) and BFT traits (Table 2). The change from glycine (GGA) to arginine (CGA) in exon 25 likely affects the function of GRIP1. To assess the importance of this SNP, we conducted multiple protein sequence alignment analysis [24] using human (ENSP00000381098), horse (ENSECAP00000006920), dog (ENSCAFP00000000554), cattle (XP_002687693.1), and pig (ENSSSCP00000000508, reference sequence) proteins, as well as our KNP sequences (Figure 2). The result showed that the nucleotide involved in the c.3316 C > G (R1106G) missense mutation is highly conserved among these species, providing further evidence for the putative functional importance of this SNP. However, any effect of the missense mutation (c.3316 C > G [R1106G]) on BFT traits must be validated in other independent

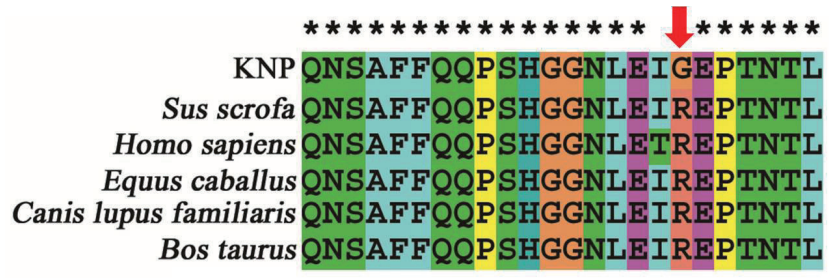

Figure 2. Multiple sequence alignment of glutamate receptor-interacting protein 1 (GRIP1) from different species. The protein sequences were aligned with Clustal X. The arrow indicates the position of c.3316 C>G (R1106G). populations prior to commercial application.

Haplo-genotype effects of the two SNPs on BFT traits are shown in Table 3. A total of seven haplo-genotypes were identified, and these haplo-genotypes exhibit different effects. The association results showed that the haplo-genotypes significantly affected BFT traits (BF_tho4_5, BF_tho11_12, and BF_tho_lum).

In this study, porcine GRIP1 was evaluated as positional candidate gene for BFT traits. We screened the GRIP1 exon regions to identify polymorphic DNA markers, and two SNPs (c.2442 $\mathrm{T}>\mathrm{C}, \mathrm{c} .3316 \mathrm{C}>\mathrm{G}[\mathrm{R} 1106 \mathrm{G}]$ ) were found to significantly affect BFT traits in an $\mathrm{F}_{2}$ intercross population between Landrace and KNP. GRIP1 plays an important role in transmission in GABAergic and glutamatergic synapses [16,17]. After validating its functional implications in obesity related traits and this positive associations in other independent populations, the markers in GRIP1 can be used for the selection of individuals with desirable BFT traits and can contribute the understanding genetic structure of BFT traits.

\section{CONFLICT OF INTEREST}

We certify that there is no conflict of interest with any financial organization regarding the material discussed in the manuscript.

Table 3. Genotypic effects of the exonic variants SNP markers on the BFT traits

\begin{tabular}{|c|c|c|c|c|c|c|}
\hline \multirow{2}{*}{$\begin{array}{l}\text { Haplo-genotype } \\
\text { (No. of head) }\end{array}$} & \multicolumn{6}{|c|}{ Traits } \\
\hline & BF_tho4_5 & $\mathrm{p}$-value & BF_tho11_12 & $\mathrm{p}$-value & BF_tho_lum & $p$-value \\
\hline ht1*ht1(167) & $35.14 \pm 0.98$ & $6.52 \times 10^{-5}$ & $28.50 \pm 0.96$ & $1.54 \times 10^{-5}$ & $28.09 \pm 0.92$ & $3.42 \times 10^{-13}$ \\
\hline$h+1{ }^{*} h t 2(39)$ & $32.67 \pm 1.27$ & & $27.02 \pm 1.23$ & & $26.29 \pm 1.17$ & \\
\hline$h t 1 * h t 3(23)$ & $32.86 \pm 1.47$ & & $27.44 \pm 1.41$ & & $27.90 \pm 1.35$ & \\
\hline ht1*ht4(494) & $34.26 \pm 0.90$ & & $27.69 \pm 0.90$ & & $27.20 \pm 0.85$ & \\
\hline ht2*ht4(57) & $32.47 \pm 1.15$ & & $25.54 \pm 1.12$ & & $24.61 \pm 1.06$ & \\
\hline ht3*ht4(11) & $29.70 \pm 1.86$ & & $23.69 \pm 1.77$ & & $23.33 \pm 1.69$ & \\
\hline$h t 4 * h t 4(311)$ & $33.13 \pm 0.93$ & & $26.49 \pm 0.92$ & & $25.01 \pm 0.88$ & \\
\hline
\end{tabular}

SNP, single nucleotide polymorphism; BFT, backfat thickness.

${ }^{1)}$ The haplotype effects were estimated using a mixed effects model.

${ }^{2)}$ The frequency of ht1 (allele C of c.3316C > G [R1106G] and allele A of c.2442T >C) was 0.403, ht2 (allele C of c.3316C > G [R1106G] and allele G of c.2442T >C) was 0.044 and ht3 (allele $\mathrm{G}$ of c.3316C > G [R1106G] and allele A of c.2442T >C) was 0.015 and ht4 (allele $G$ of c.3316C > G [R1106G] and allele G of c.2442T >C) was 0.538. 


\section{ACKNOWLEDGMENTS}

This work was carried out with the support of "Cooperative Research Program for Agriculture Science and Technology Development (Project No.PJ009971032017)" Rural Development Administration, Republic of Korea. Please check again!

\section{REFERENCES}

1.Lee KT, Byun MJ, Kang KS, et al. Neuronal genes for subcutaneous fat thickness in human and pig are identified by local genomic sequencing and combined SNP association study. PLOS ONE 2011;6:e16356.

2.Fujii J, Otsu K, Zorzato F, et al. Identification of a mutation in porcine ryanodine receptor associated with malignant hyperthermia. Science 1991;253:448-51.

3.Zhang YH, Dai LS, Ma TH, et al. Association of T1740C polymorphism of L-FABP with meat quality traits in Junmu No. 1 white swine. Genet Mol Res 2013;12:235-41.

4.Fontanesi L, Scotti E, Buttazzoni L, Davoli R, Russo V. The porcine fat mass and obesity associated (FTO) gene is associated with fat deposition in Italian Duroc pigs. Anim Genet 2009;40:90-3.

5.Hernández-Sánchez J, Visscher P, Plastow G, Haley C. Candidate gene analysis for quantitative traits using the transmission disequilibrium test: the example of the melanocortin 4-receptor in pigs. Genetics 2003;164:637-44.

6.Kang K, Cahyadi M, Park HB, et al. Association of variation in the mc4r gene with meat quality traits in a commercial pig population. J Fac Agric Kyushu Univ 2015;60:113-8.

7.Yu TP, Tuggle CK, Schmitz CB, Rothschild MF. Association of PIT1 polymorphisms with growth and carcass traits in pigs. J Anim Sci 1995;73:1282-8.

8.Stančeková K, Vašíček D, Peškovičová D, Bulla J, Kúbek A. Effect of genetic variability of the porcine pituitary-specific transcription factor (PIT-1) on carcas traits in pigs. Anim Genet 1999;30:313-5.

9.Song C, Gao B, Teng Y, et al. MspI polymorphisms in the 3rd intron of the swine POU1F1 gene and their associations with growth performance. J Appl Genet 2005;46:285-9.

10. Milan, D, Jeon JT, Looft C, et al. A mutation in PRKAG3 associated with excess glycogen content in pig skeletal muscle. Science 2000;288: 1248-51.
11. Ciobanu D, Bastiaansen J, Malek M, et al. Evidence for new alleles in the protein kinase adenosine monophosphate-activated $\gamma 3$-subunit gene associated with low glycogen content in pig skeletal muscle and improved meat quality. Genetics 2001;159:1151-62.

12. Ryan MT, Hamill RM, O'Halloran AM, et al. SNP variation in the promoter of the PRKAG3 gene and association with meat quality traits in pig. BMC Genet 2012;13:66.

13. Yoo CK, Lim HT, Han SH, et al. QTL analysis of back fat thickness and carcass $\mathrm{pH}$ in an $\mathrm{F}_{2}$ intercross between Landrace and Korean native pigs. Mol Biol Rep 2012;39:8327-33.

14. Brückner K, Labrador JP, Scheiffele P, et al. EphrinB ligands recruit GRIP family PDZ adaptor proteins into raft membrane microdomains. Neuron 1999;22:511-24.

15. Setou M, Seog DH, Tanaka Y, et al. Glutamate-receptor-interacting protein GRIP1 directly steers kinesin to dendrites. Nature 2002;417: 83-7.

16. Dong H, Zhang P, Song I, et al. Characterization of the glutamate receptor-interacting proteins GRIP1 and GRIP2. J Neurosci 1999;19: 6930-41.

17. Delgado T. Glutamate and GABA in appetite regulation. Front Endocrinol 2013;4.

18. Wu Q, Clark MS, Palmiter RD. Deciphering a neuronal circuit that mediates appetite. Nature 2012;483:594-7.

19. Cho IC, Park HB, Yoo CK, et al. QTL analysis of white blood cell, platelet and red blood cell-related traits in an $\mathrm{F}_{2}$ intercross between Landrace and Korean native pigs. Anim Genet 2011;42:621-6.

20. Henderson C. Application of linear model in animal breeding. Guelph, Canada: University of Guelph, 1984.

21. Gilmour AR, Thompson R, Cullis BR. Average information REML: an efficient algorithm for variance parameter estimation in linear mixed models. Biometrics 1995;51:1440-50.

22. Li J, Jiang T. Computing the minimum recombinant haplotype configuration from incomplete genotype data on a pedigree by integer linear programming. J Comput Biol 2005;12:719-39.

23. Liu SJ, Cull-Candy SG. Subunit interaction with PICK and GRIP controls $\mathrm{Ca}^{2+}$ permeability of AMPARs at cerebellar synapses. Nat Neurosci 2005;8:768-75.

24. Kohli DK, Bachhawat AK. CLOURE: Clustal output reformatter, a program for reformatting ClustalX/ClustalW outputs for SNP analysis and molecular systematics. Nucleic Acids Res 2003;31:3501-2. 